\title{
Hamburger Nuklearmedizin-Tage 2020
}

Die 27. Hamburger Nuklearmedizin-Tage, die sich insbesondere an Facharztkandidaten und Fachärzte richten, finden vom 3. bis 5. Juni 2020 in Hamburg statt. Sie werden erneut unter der bewährten wissenschaftlichen Leitung von Herrn Prof. Dr. Matthias Schmidt (Köln) veranstaltet.

Die Hamburger Nuklearmedizin-Tage erfreuen sich durch ihren Seminarcharakter seit
Jahren außerordentlich hoher Beliebtheit. Klinisch langjährig erfahrene Referenten haben sich zum Ziel gesetzt, praxisrelevantes Facharztwissen lebendig zu vermitteln. Die Zielgruppen sind damit v.a. Kolleginnen und Kollegen in der fortgeschrittenen Facharztausbildung, die den Kurs zur Vorbereitung auf die Facharztprüfung nutzen können oder den Kurs als „NUK-Refresher“ schätzen. Zahlreiche Fallbeispiele und die Möglichkeit des jederzeitigen Fragens bieten ein höchstes Maß an Fortbildungseffizienz.

Weitere Informationen zu den Hamburger Nuklearmedizin-Tagen finden Sie im Veranstaltungskalender auf der DGN-Homepage www.nuklearmedizin.de. Die Online-Anmeldung steht Ihnen Anfang 2020 zur Verfügung. 